\title{
Remarks on hard Lefschetz conjectures on Chow groups
}

\author{
Baohua Fu
}

October 26, 2018

\begin{abstract}
We propose two conjectures of Hard Lefschetz type on Chow groups and prove them for some special cases. For abelian varieties, we shall show they are equivalent to well-known conjectures of Beauville and Murre.
\end{abstract}

\section{Introduction}

Let $X$ be a smooth complex projective variety of dimension $n$ and $D$ an ample divisor on $X$. We denote by $C H^{p}(X)$ the Chow group of codimension $p$ algebraic cycles on $X$ with rational coefficients. Let $c l: C H^{p}(X) \rightarrow H^{2 p}(X, \mathbb{Q})$ be the class map. We have the following commutative diagram:

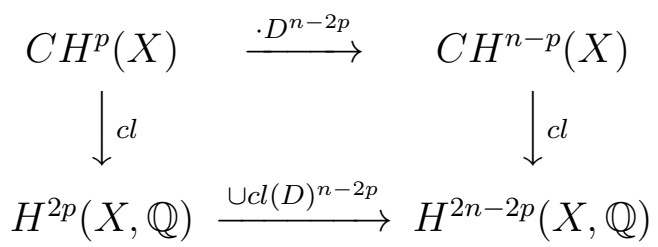

The hard Lefschetz theorem on cohomology says that the bottom map $\cup c l(D)^{n-2 p}$ is an isomorphism. Note that the map $\cdot D^{n-2 p}$ is in general not surjective, since by a famous result of Mumford, $C H^{n}(X)$ could be very complicated while $C H^{0}(X) \simeq \mathbb{Q}$. We propose in this paper the following conjecture on Chow groups.

Conjecture 1. If $n \geq 2 p$, the map $C H^{p}(X) \stackrel{\cdot D^{n-2 p}}{\longrightarrow} C H^{n-p}(X)$ is injective. 
For an abelian variety defined over a finite field, it is proven by Soulé ([S]) that the above map is an isomorphism. Another conjecture of Hard Lefschetz type that we will discuss is the following:

Conjecture 2. If $n \geq 2 p-1$, the map $C H^{p}(X)_{h o m} \stackrel{\cdot D^{n-2 p+1}}{\longrightarrow} C H^{n-p+1}(X)_{h o m}$ is injective, where $C H^{p}(X)_{h o m}=\operatorname{ker}\left(C H^{p}(X) \stackrel{c l}{\rightarrow} H^{2 p}(X)\right)$.

Convention: We will say Conjecture 1 or 2 for a pair $(X, D)$ or a triple $(X, D, p)$ when we want to emphasize the special $D$ or $p$.

As easily seen, Conjecture 2 implies Conjecture 1. We shall prove in the next section that both conjectures follow from the standard conjecture of Lefschetz type $([\mathrm{G}]$ ) and Bloch-Beilinson's conjecture (see for example [J]) on the existence of a functorial filtration on Chow groups.

In the third section, we prove both conjectures for $p=1$, which shows that Conjecture 1 (resp. Conjecture 2) holds when $\operatorname{dim}(X) \leq 4$ (resp. $\operatorname{dim}(X) \leq$ $3)$. With the help of [B-S], some partial results for $p=2$ are obtained in this section, which shows that Conjecture 1 (resp. Conjecture 2) holds for rationally connected manifolds of dimension $\leq 6$ (resp. $\leq 5)$.

In section 4, we first prove that if Conjecture1(resp. Conjecture 2) holds for $(X, D)$, then it also holds for $\left(Z, f^{*}(D)+m E x c(f)\right)$, where $f: Z \rightarrow X$ is the blow-up of $X$ along a smooth subvariety of dimension $\leq 2($ resp. $\leq 1)$ and $m<0$ is a rational number such that $f^{*}(D)+m E x c(f)$ is ample. Then we prove that if Conjecture 1 (resp. Conjecture 21) holds for $X$, then it holds for $X \times \mathbb{P}^{m}$. Also we prove Conjecture 1 (resp. Conjecture 2) holds for projective bundles over smooth varieties of dimension $\leq 2($ resp. $\leq 1)$. Together with the examples of small dimension, these two properties provide many examples supporting Conjecture 1 and Conjecture 2.

In the fifth section, we reveal the relationship between Conjecture 1 and Beauville's conjecture ([B1], [B2]). More precisely, we prove that Beauville's conjecture is equivalent to Conjecture 1 for abelian varieties or to Conjecture 1 for symmetric products $\left(C^{(k)}, z_{k}\right)$ of curves, where $z_{k}$ is the ample divisor $C^{(k-1)}+p t$. Finally we prove in the last section that for abelian varieties, Murre's conjectures (see [M]) are equivalent to Conjecture 2. In particular, this proves Conjecture 1 (resp. Conjecture 2) for $\left(C^{(k)}, z_{k}\right)$ with $g(C) \leq 4$ (resp. $g(C) \leq 3$ ).

Acknowledgements: It is my pleasure to thank A. Beauville, A. Collino and C. Voisin for their suggestions and corrections to a previous version. 
Part of this work has been done during my visits to FuDan University and to KIAS. I'd like to thank M. Chen and J.-M. Hwang for the invitations and for the helpful discussions.

\section{Bloch-Beilinson's conjecture}

Recall that the Bloch-Beilinson conjecture (see for example [J]) asserts the existence of a decreasing filtration $F^{i} C H^{p}(X)$ for any smooth projective variety $X$, satisfying the following properties:

(i) $F^{0} C H^{p}(X)=C H^{p}(X), F^{1} C H^{p}(X)=C H^{p}(X)_{h o m}$;

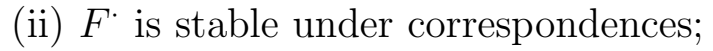

(iii) If $\Gamma \in C H^{k}(X \times Y)$ induces the zero map from $H^{2 l-i}(X, \mathbb{Q})$ to $\left.H^{2 k+2 l-2 n-i}(Y, \mathbb{Q})\right)$, then so is the map $G r_{F}^{i} \cdot \Gamma: G r_{F}^{i} \cdot C H^{l}(X) \rightarrow G r_{F}^{i} C H^{k+l-n}(Y)$, where $n=\operatorname{dim}(X)$;

(iv) $F^{p+1} C H^{p}(X)=0$.

Proposition 1. Assume the Bloch-Beilinson conjecture and the standard conjecture of Lefschetz type([G]), then Conjecture 1 and Conjecture $[$ hold.

Proof. The proof here is inspired by [V2]. Up to replacing $D$ by a higher multiple, we may assume that $D$ is a smooth irreducible divisor on $X$ such that $Y:=D^{n-i}$ is again smooth and irreducible. Let $\iota: Y \rightarrow X$ be the natural inclusion and $\Gamma_{\iota}$ its graph. Then the map $\cdot D^{n-i}$ is induced by the cycle $\Gamma:=\Gamma_{\iota} \circ \Gamma_{\iota}^{t} \in C H^{2 n-i}(X \times X)$. By the standard conjecture of Lefschetz type, there exists an algebraic cycle $\Gamma^{\prime} \in C H^{i}(X \times X)$ such that $\Gamma_{*}^{\prime} \circ \Gamma_{*}=$ $I d: H^{i}(X, \mathbb{Q}) \rightarrow H^{i}(X, \mathbb{Q})$.

By Bloch-Beilinson conjecture (iii), we have $\Gamma_{*}^{\prime} \circ \Gamma_{*}=I d: G r_{F}^{v} C H^{p}(X) \rightarrow$ $G r_{F}^{v} C H^{p}(X)$ for $2 p-v=i$. In particular, $\Gamma_{*}=\cdot D^{n-i}: G r_{F}^{v} \cdot C H^{p}(X) \rightarrow$ $G r_{F}^{v} C H^{p+n-i}(X)$ is injective for $2 p-v=i$. This implies that the map

$$
G r_{F}^{v} \cdot C H^{p}(X) \stackrel{\cdot D^{n-2 p}}{\longrightarrow} G r_{F}^{v} \cdot C H^{n-p}(X)
$$

is injective for all $v \geq 0$. By statement (iv) in Bloch-Beilinson conjecture, Conjecture 1 holds.

For Conjecture 2, the same argument shows that $G_{F}^{v} \cdot C H^{p}(X) \stackrel{\cdot D^{n-2 p+1}}{\longrightarrow}$ $G r_{F}^{v} C H^{n-p+1}(X)$ is injective for all $v \geq 1$. Now the conclusion follows from statement (i) in Bloch-Beilinson conjecture. 
As is well-known, the Bloch-Beilinson conjecture is fundamental to understand Chow groups, while it is extremely hard to prove. Hopefully, the two conjectures of Hard Lefschetz type could be easier to be checked for various examples.

\section{Small codimension}

The following Lemma is immediate from the Hard Lefschetz theorem on cohomologies.

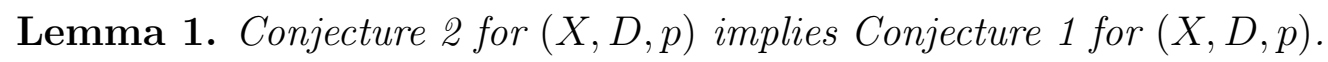

We prove in the following that Conjecture 1 and 2 are true for $p=1$, which could be already known. This implies that Conjecture 1 holds when $\operatorname{dim}(X) \leq 4$ and Conjecture 2 holds when $\operatorname{dim}(X) \leq 3$.

Proposition 2. Let $X$ be a smooth projective variety of dimension $n$ and $D$ an ample divisor. Then $C H^{1}(X) \stackrel{\cdot D^{n-2}}{\longrightarrow} C H_{1}(X)$ and $C H^{1}(X)_{\text {hom }} \stackrel{\cdot D^{n-1}}{\longrightarrow}$ $C H_{0}(X)_{\text {hom }}$ are injective.

Proof. Consider the Albanese map $C H_{0}(X, \mathbb{Z})_{\text {hom }} \stackrel{a l b}{\longrightarrow} A l b(X)$ and the composition $a l b \circ\left(D^{n-1} \cdot\right): P i c^{0}(X) \rightarrow A l b(X)$. By the Hard Lefschetz theorem, the map $H^{1}(X, \mathbb{Q}) \stackrel{\cup c l(D)^{n-1}}{\longrightarrow} H^{2 n-1}(X, \mathbb{Q})$ is an isomorphism, which implies that the map alb $\circ\left(D^{n-1} \cdot\right)$ is an isogeny. In particular, it is injective after tensor product with $\mathbb{Q}$, which gives the second statement. The first one follows from Lemma 1 .

Here are some consequences of this.

Corollary 1. Let $C$ be a smooth projective curve of genus $g$ and $J$ its Jacobian. Fix one point on $C$ and consider the associated Abel-Jacobi map: $C \stackrel{\iota}{\rightarrow} J$. Then the induced map $\iota_{*}: \mathrm{CH}_{0}(C)_{\text {hom }} \rightarrow C H_{0}(J)_{\text {hom }}$ is injective.

Proof. Note that $\iota^{*}: \operatorname{Pic}^{0}(J) \rightarrow \operatorname{Pic}^{0}(C)$ is surjective. Let $\alpha \in C H_{0}(C)_{h o m}$ with $\iota_{*}(\alpha)=0$. Then there exists an element $\beta \in C H^{1}(J)_{\text {hom }}$ such that $\alpha=$ $\iota^{*} \beta$, then $0=\iota_{*}(\alpha)=\iota_{*}\left(\iota^{*} \beta\right)=\beta \cdot \iota_{*}(C)$. By [B2], we have a decomposition $\iota_{*}(C)=\sum_{s=0}^{g-1} C_{(s)}$, where $C_{(s)} \in C H_{(s)}^{g-1}(J)$. As $\beta$ is in $C H_{(1)}^{1}(J)$, we obtain that $\beta \cdot C_{(0)}=0$. Let $\Theta$ be a symmetric theta divisor. We have $C_{(0)}=\frac{\Theta^{g-1}}{(g-1) !}$. As a consequence, $\beta \cdot \Theta^{g-1}=0$, which gives $\beta=0$ by the previous Proposition, so $\alpha=0$. 
Remark 1. As pointed out by C. Voisin, one can prove this corollary by noting that $C H_{0}(C)_{h o m} \rightarrow A l b(C)$ is injective and $A l b(C)=A l b(J(C))$.

Corollary 2. Let $\iota: C \rightarrow S$ be the inclusion of an ample divisor in a smooth projective surface $S$ such that $q(S)=g(C)$. Then the map $\iota_{*}: C H_{0}(C)_{h o m} \rightarrow$ $\mathrm{CH}_{0}(\mathrm{C})_{\text {hom }}$ is injective.

Proof. Consider the map $\iota^{*}: P i c^{0}(S) \rightarrow P i c^{0}(C)$. If $\iota^{*}(\alpha)=0$ for some $\alpha \in P i c^{0}(S)$, then $\alpha \cdot C=0$, which implies by the previous proposition that $m \alpha=0$ for some $m>0$, i.e. $\alpha$ is a torsion point. The condition $q(S)=g(C)$ implies then $\iota^{*}$ is an isogeny, which is in particular surjective. Now a similar argument as in the proof of the previous Lemma concludes the proof.

Remark 2. (i). In [V1], a similar but much more difficult result is proved for general hyperplane sections of a degree $d \geq 5$ surface $S$ in $\mathbb{P}^{3}$. However, our result cannot be applied here, since the condition $q(S)=g(C)$ is never satisfied in this case.

(ii). Examples of pairs $(S, C)$ such that $C$ is ample and $g(C)=q(S)$

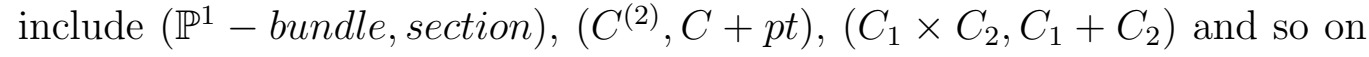
(for a classification of such pairs see $[\mathrm{F}]$ ).

Now we study the case of $p=2$, where the main difficulty is that $C H^{2}(X)$ is in general "infinite-dimensional".

Proposition 3. Let $X^{n}$ be a smooth projective variety and $V \subset X$ a closed subvariety of dimension $\leq 1$ such that $C H_{0}(V) \rightarrow C H_{0}(X)$ is surjective. Then Conjecture 1 and Conjecture 1 hold for $\mathrm{CH}^{2}(X)$.

Proof. Let $J^{2}(X)$ be the second intermediate Jacobian of $X$. By [B-S], the hypothesis implies that $C H^{2}(X)_{h o m} \rightarrow J^{2}(X) \otimes \mathbb{Q}$ is injective. By the Hard Lefschetz theorem, $\cup \operatorname{cl}\left(D^{n-3}\right): H^{3}(X, \mathbb{Q}) \rightarrow H^{2 n-3}(X, \mathbb{Q})$ is an isomorphism for any ample divisor $D$ on $X$, which implies that the map $\cdot D^{n-3}: J^{2}(X) \rightarrow$ $J^{n-1}(X)$ has finite kernel. As in the proof of Proposition 2, this implies Conjecture 2,

Note that $\mathrm{CH}_{0}$ is birationally invariant, and $C H_{0}(F)=\mathbb{Q}$ for any rationally connected manifold $F$. The previous proposition gives the following result.

Corollary 3. Conjecture 1 (resp. Conjecture 圆) holds for smooth projective varieties of dimension $\leq 6$ (resp. $\leq 5)$ which are fibrations over a smooth curve with general fibers being rationally connected. 


\section{Blow-ups and projective bundles}

Now we study the behavior of both conjectures under blow-ups. Let $\iota: Y \rightarrow$ $X$ be the inclusion of a smooth subvariety $Y$ of codimension $r+1 \geq 2$ in a smooth projective variety $X^{n}$. Write $d:=\operatorname{dim} Y=n-r-1$. We denote by $f: Z \rightarrow X$ the blow-up of $X$ along $Y$ and $E$ the exceptional divisor. We use the following notations for morphisms:

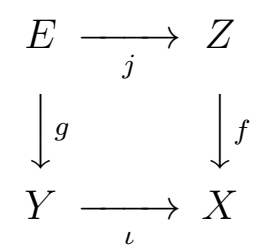

Proposition 4. Assume Conjecture 1 (resp. Conjecture 2) holds for $(X, L)$ with $L$ an ample divisor on $X$, then Conjecture 1 (resp. Conjecture Q2) holds for $\left(Z, f^{*}(L)+m E, p\right)$ with $p \geq d$ (resp. $\left.p \geq d-1\right)$, where $m<0$ is a rational number such that $f^{*}(L)+m E$ is ample on $Z$.

Proof. We will give details for the case of Conjecture 1, while the proof of the case of Conjecture 2 is completely similar. As the case $p=1$ has been proved in the last section, we may assume that $p \geq 2$.

Take an element $\alpha \in C H^{p}(Z)$. By the blow-up formula for Chow groups, we can write $\alpha=f^{*}(x)+\sum_{i=0}^{r} j_{*}\left(h^{p-1-i} g^{*} y_{i}\right)$, where $x \in C H^{p}(X), y_{i} \in$ $C H^{i}(Y)$ and $h=c_{1}\left(\mathcal{O}_{g}(1)\right)$. Assume now $\left(f^{*} L+m E\right)^{n-2 p} \alpha=0$. By Hard Lefschetz theorem for cohomologies, one has $y_{0}=0$. This gives

$$
\begin{aligned}
f^{*}\left(L^{n-2 p} x\right)+ & f^{*} L^{n-2 p} j_{*}\left(\sum_{i=1}^{r} h^{p-1-i} g^{*} y_{i}\right)+\sum_{l=1}^{n-2 p}\left(\begin{array}{c}
n-2 p \\
l
\end{array}\right) m^{l} f^{*}\left(L^{n-2 p-l} x\right) E^{l} \\
& +j_{*}\left(\sum_{i=1}^{r} h^{p-1-i} g^{*} y_{i}\right) \sum_{l=1}^{n-2 p}\left(\begin{array}{c}
n-2 p \\
l
\end{array}\right) m^{l} f^{*}\left(L^{n-2 p-l}\right) E^{l}=0 .
\end{aligned}
$$

Note that $l \leq n-2 p \leq r-1$, we have $f_{*}\left(E^{l}\right)=0$. As $l+p-1-$ $i \leq n-p-2 \leq n-d-2=r-1$, we obtain $f_{*}\left(j_{*}\left(h^{p-1-i} g^{*} y_{i}\right) E^{l}\right)=$ $(-1)^{l} f_{*} j_{*}\left(h^{l+p-1-i} g^{*} y_{i}\right)=0$. Apply $f_{*}$ to the above equation, then we obtain $L^{n-2 p} x=0$. By hypothesis, this implies that $x=0$. Now the above equation is equivalent to the following (use the projection formula and $j^{*} E=-h$ ):

$j_{*}\left(\sum_{i=1}^{r} h^{p-1-i} g^{*}\left(y_{i} \iota^{*} L^{n-2 p}\right)+\sum_{i=1}^{r} \sum_{l=1}^{n-2 p}\left(\begin{array}{c}n-2 p \\ l\end{array}\right)(-m)^{l} h^{l+p-1-i} g^{*}\left(y_{i} \iota^{*} L^{n-2 p-l}\right)\right)=0$ 
Applying successively $g_{*}\left(h^{p-d+k} j^{*}(\cdot)\right)(k=0,1, \cdots)$ to the above equation and using the relations $j^{*} j_{*}=-h$ and $g_{*}\left(h^{i} g^{*}(y)\right)=\delta_{i, r} y$ for $i \leq r$ and $y \in C H(Y)$, we obtain that $y_{1}=y_{2}=\cdots=y_{d}=0$.

Corollary 4. Assume Conj. 1 (resp. Conj. 2) holds for $(X, D)$. Let $f$ : $Z \rightarrow X$ be the blow-up of $X$ along a smooth center of dimension $\leq 2$ (resp. $\leq 1)$ and $m<0$ such that $D^{\prime}:=f^{*} D+m E x c(f)$ is ample. Then Conj. 1 (resp. Conj. 2) holds for $\left(Z, D^{\prime}\right)$.

Remark 3. When $f: Z \rightarrow X$ is the blow-up of a point, then one may use the Nakai-Moishezon criteria of ampleness to show that if $f^{*} D+m E x c(f)$ is ample on $Z$, then $D$ is ample on $X$. In this case, Conj. 1 (resp. Conj. 2) holds for $X$ implies that it holds also for $Z$.

The following proposition can be proved in a similar way.

Proposition 5. (1) Assume Conjecture 1 (resp. Conjecture 2) holds for X, then it also holds for the product $X \times \mathbb{P}^{m}$.

(2) Conjecture 1 (resp. Conjecture Q) holds for projective bundles over a smooth projective variety of dimension $\leq 2$ (resp. $\leq 1)$.

\section{Beauville's conjecture}

Let $A$ be a $g$-dimensional complex abelian variety. By [B2], we have a decomposition of the Chow groups of $A$ as follows: $C H^{p}(A)=\oplus_{s=p-g}^{p} C H_{(s)}^{p}(A)$, where $C H_{(s)}^{p}(A)$ consists of classes $\alpha \in C H^{p}(A)$ such that for any $k \in \mathbb{Z}$, we have $\mathbf{k}^{*} \alpha=k^{2 p-s} \alpha$, where $\mathbf{k}: A \rightarrow A$ is the multiplication by $k$. It was conjectured in [B1] and [B2] that $C H_{(s)}^{p}(A)=0$ if $s<0$, which has been proved for $p \in\{0,1, g-2, g-1, g\}$ ([B1]). Since then, there has appeared several equivalent formulations of Beauville's conjecture, for example, in [K], it was shown that Beauville's conjecture is equivalent to the hypercube conjecture for abelian varieties. Unfortunately, despite its importance, very little progress has been made (neither for these reformulations) during the last twenty years.

In [K-V], Kimura and Vistoli proved that Beauville's conjecture is equivalent to the strong stability conjecture on Chow groups of symmetric products of curves. To state it, we need several notations.

Let $C$ be a smooth projective connected curve and $c_{0} \in C$ a fixed point. The $n$-th symmetric product of $C$ will be denoted by $C^{(n)}$. Let $\phi_{n}: C^{(n-1)} \rightarrow$ 
$C^{(n)}$ be the addition of the point $c_{0}$. We will denote by $z_{n} \in C H^{1}\left(C^{(n)}\right)$ the class of the divisor $\phi_{n}\left(C^{(n-1)}\right)$ and we set $z:=z_{2 g-1}$. The Jacobian of $C$ will be denoted by $J(C)$. The strong stability conjecture of [K-V] asserts that for all $n \geq 2 p+1$, the map $\phi_{n}^{*}: C H^{p}\left(C^{(n)}\right) \rightarrow C H^{p}\left(C^{(n-1)}\right)$ is an isomorphism;

In $[\mathrm{P}$, Paranjape conjectured (which goes back to [H]) the following analogue to the weak Lefschetz theorem: if $\iota: Y \hookrightarrow X$ is the inclusion of a smooth ample divisor in a smooth projective variety, then $\iota^{*}: C H^{p}(X) \rightarrow$ $C H^{p}(Y)$ is an isomorphism for $p<\operatorname{dim}(Y) / 2$. In relation with our conjectures here, we have

Lemma 2. If Conjecture 1 holds for $X$, then for any smooth ample divisor $\iota: Y \hookrightarrow X$, the map $\iota^{*}: C H^{p}(X) \rightarrow C H^{p}(Y)$ is injective for $p \leq \operatorname{dim}(Y) / 2$.

Proof. It suffices to notice that $\iota_{*}\left(\iota^{*} \bullet\right)=\bullet Y: C H^{p}(X) \rightarrow C H^{p+1}(X)$, which is injective if $\operatorname{dim}(X)-2 p \geq 1$.

Proposition 6. If Conjecture 1 holds for all symmetric products $\left(C^{(n)}, z_{n}\right)$ of a curve $C$, then the strong stability conjecture holds for $C$.

Proof. It was shown in [C] that $\phi_{n}^{*}$ is always surjective, thus we just need to prove it is injective. This follows from the previous lemma, since $z_{n}$ is an ample divisor.

It turns out that the same conclusion holds if one only assumes Conjecture 1 for $\left(C^{(2 g-1)}, z\right)$, as shown by the following:

Proposition 7. If Conjecture 1 holds for $\left(C^{(2 g-1)}, z\right)$, then the strong stability conjecture holds for $C$. Furthermore Conjecture 1 for $\left(C^{(2 g-1)}, z\right)$ is equivalent to Beauville's conjecture for $J(C)$.

Proof. By Prop. 2.9 (a) $[\mathrm{K}-\mathrm{V}]$, the strong stability conjecture is true if $n>2 g-1$. We may assume in the following that $2 g-1 \geq n$. Let $i_{n}: C^{(n)} \rightarrow$ $C^{(2 g-1)}$ be the composition of additions of the point $c_{0}$. By [C], the morphism $\left(i_{n}\right)_{*}: C H\left(C^{(n)}\right) \rightarrow C H\left(C^{(2 g-1)}\right)$ is injective and $\left(i_{n}\right)_{*}\left(z_{n}^{k}\right)=z^{2 g-1-n+k}$.

Note that we just need to prove the injectivity of $\phi_{n}^{*}$. Let $\alpha \in C H^{p}\left(C^{(n)}\right)$, then

$$
\begin{aligned}
\phi_{n}^{*}(\alpha)=0 & \Leftrightarrow\left(i_{n-1}\right)_{*}\left(\phi_{n}^{*} \alpha\right)=0 \Leftrightarrow\left(i_{n}\right)_{*}\left(\phi_{n}\right)_{*}\left(\phi_{n}^{*} \alpha\right)=0 \\
& \Leftrightarrow\left(i_{n}\right)_{*}\left(\alpha \cdot\left(\phi_{n}\right)_{*}\left[C^{(n-1)}\right]\right)=\left(i_{n}\right)_{*}\left(\alpha z_{n}\right)=0 .
\end{aligned}
$$


Let $p_{n}: C^{(n)} \rightarrow J(C)$ be the morphism $D \mapsto \mathcal{O}_{C}\left(D-n c_{0}\right)$, then $p_{n}=$ $p_{2 g-1} \circ i_{n}$. By [C], we can write $\alpha=\sum_{j} p_{n}^{*}\left(a_{j}\right) z_{n}^{p-j}$ with $a_{j} \in C H^{j}(J(C))$. We have

$$
\begin{aligned}
\left(i_{n}\right)_{*}\left(\alpha z_{n}\right) & =\sum_{j}\left(i_{n}\right)_{*}\left(i_{n}^{*} p_{2 g-1}^{*}\left(a_{j}\right) \cdot z_{n}^{p-j+1}\right)=\sum_{j} p_{2 g-1}^{*}\left(a_{j}\right) \cdot\left(i_{n}\right)_{*}\left(z_{n}^{p-j+1}\right) \\
& =\sum_{j} p_{2 g-1}^{*}\left(a_{j}\right) \cdot z^{2 g-n+p-j}=\left(\sum_{j} p_{2 g-1}^{*}\left(a_{j}\right) z^{p-j}\right) z^{2 g-n} .
\end{aligned}
$$

If $\phi_{n}^{*} \alpha=0$, then $\left(\sum_{j} p_{2 g-1}^{*}\left(a_{j}\right) z^{p-j}\right) z^{2 g-n}=0$. Note that $z$ is ample and $2 g-1-2 p \geq 2 g-n$. We have $\left(\sum_{j} p_{2 g-1}^{*}\left(a_{j}\right) z^{p-j}\right) z^{2 g-1-2 p}=0$. As Conjecture 1 holds for $\left(C^{(2 g-1)}, z\right)$, we get $\sum_{j} p_{2 g-1}^{*}\left(a_{j}\right) z^{p-j}=0$, which gives $\alpha=i_{n}^{*}\left(\sum_{j} p_{2 g-1}^{*}\left(a_{j}\right) z^{p-j}\right)=0$.

The second statement follows from Prop. 2.18 in [K-V].

Now we consider Conjecture 1 for $\left(C^{(2 g-1)}, z\right)$. Recall that the natural map $p_{k}: C^{(k)} \rightarrow J(C)$ is birational onto its image when $k \leq g$. In this case, we set $w_{k}=p_{g-k}\left(C^{(g-k)}\right) \in C H^{k}(J(C))$ and $v_{k}=(-1)^{k}\left(T_{c}\right)_{*}(-1)_{*} w_{k}$, where $T_{c}$ is the translation and $c$ is the image of the canonical divisor on $C$ in $J(C)$. Recall that the map $C^{(2 g-1)} \rightarrow J(C)$ is a projective bundle $\mathbb{P}(F) \rightarrow J(C)$ and the total Chern class of $F$ was computed in [Ma]: $c(F)=\sum_{k=0}^{g} v_{k}$. In particular, the Chow group $C H\left(C^{(2 g-1)}\right)$ is given by $C H(J(C))[z]$ (here we identify $C H(J(C))$ with its image under $\left.p_{2 g-1}^{*}\right)$, and the minimal equation of $z$ is $\alpha:=\sum_{k=0}^{g} v_{k} z^{g-k}=0$.

Let $p$ be a non-negative integer such that $2 g-1 \geq 2 p+1$ and $k:=$ $g-p-1$. Assume first $k \leq p$. Let $y=\sum_{i=0}^{p} y_{i} z^{p-i} \in C H^{p}\left(C^{(2 g-1)}\right)$ with $y_{i} \in C H^{i}(J(C))$ such that $y \cdot z^{2 g-2 p-1}=0$. Then by the Hard Lefschetz theorem, we have $y_{0}=0$ in $C H^{0}(J(C))$.

Let $a_{1}=y_{1}, \cdots, a_{k}=y_{k}-\sum_{j=1}^{k-1} a_{j} v_{k-j}$. As $y \cdot z^{2 g-2 p-1}=0$ is equivalent to $\sum_{i=1}^{p} y_{i} z^{g+k-i}=0$, by replacing powers of $z$ of degrees no less than $g$ by using the relation $z^{g}=-\left(\sum_{i=1}^{g} v_{i} z^{g-i}\right)$, we obtain a relation between $1, z, \cdots, z^{g-1}$ with coefficients in $C H(J(C))$. Thus the coefficients are zeros, which gives the following equations in $C H(J(C))$ :

$$
\text { (1) }\left\{\begin{array}{c}
a_{1} v_{p}+\cdots+a_{k} v_{p+1-k}=0 \\
\vdots \\
a_{1} v_{g-1}+\cdots+a_{k} v_{g-k}=0
\end{array}\right.
$$




$$
\text { (2) }\left\{\begin{array}{c}
y_{k+1}=a_{1} v_{k}+\cdots+a_{k} v_{1} \\
\vdots \\
y_{p}=a_{1} v_{p-1}+\cdots+a_{k} v_{p-k}
\end{array}\right.
$$

Lemma 3. Let $C$ be a smooth projective curve. Assume that the strong stability conjecture holds for $C$ with $p=g-1-l$, then Beauville's conjecture holds for $C H^{l}(J(C))$.

Proof. As in [K-V], we will denote by $C H^{p}\left(S^{\infty} C\right)$ the inverse limit $\lim _{\longleftarrow} C H^{p}\left(C^{(m)}\right)$ and $\mathcal{O}(1)$ the natural line bundle on $S^{\infty} C$. Then by Prop. 2.9 in loc. cit., we have

$$
C H^{p}\left(S^{\infty} C\right)=C H^{p}(J(C)) \oplus C H^{p-1}(J(C)) \cdot c_{1}(\mathcal{O}(1)) \oplus \cdots \oplus C H^{0}(J) \cdot c_{1}(\mathcal{O}(1))^{p} .
$$

As the strong stability conjecture holds for $p=g-1-l$, the eigenvalues of the multiplication by $N$ on $C H^{p}\left(S^{\infty} C\right)$ are $N^{0}, \cdots, N^{2(g-1-l)}$. As a consequence, for $q \leq p$, the eigenvalues of $C H^{q}(J(C))$ are $N^{q}, \cdots, N^{q+g-1-l}$. Thus we obtain that $C H_{(q-g+l)}^{q}(J(C))=0$ for $q \leq g-l-1$. By the isomorphism $C H_{(q-g+l)}^{q}(J(C)) \simeq C H_{(q-g+l)}^{l}(J(C))$ for all $q \leq g-l-1$, we obtain that $C H_{(s)}^{l}(J(C))=0$ for all $s<0$, i.e. Beauville's conjecture holds for $C H^{l}(J(C))$.

Lemma 4. Let $\Theta$ be a symmetric theta divisor on a Jacobian $J(C)$. Assume Conjecture 1 for $(J(C), \Theta)$. Then Conjecture 1 holds for $\left(C^{(2 g-1)}, z, p\right)$ such that $2 p+1 \geq g$.

Proof. Let $k:=g-p-1$. As $2 p+1 \geq g$, we have $k \leq p$ and we will use the same notations as in the discussions before Lemma 3. Let $b_{i}=$ $(-1)^{*} T_{c}^{*} a_{i}, 1 \leq i \leq k$, then by using the projection formula, equations (1) become

$$
\text { (3) }\left\{\begin{array}{c}
b_{1} w_{p}-b_{2} w_{p-1}+\cdots+(-1)^{k} b_{k} w_{p+1-k}=0 \\
\vdots \\
b_{1} w_{g-1}-b_{2} w_{g-2}+\cdots+(-1)^{k} b_{k} w_{g-k}=0
\end{array}\right.
$$

We need to prove that $b_{i}=0$ for any $i$. Assume this is not the case. Let $\left(b_{i}\right)_{(s)}$ be the component of $b_{i}$ in $C H_{(s)}^{i}(J(C))$. Let $j=\min \left\{s \mid \exists i\right.$ s.t. $\left(b_{i}\right)_{(s)} \neq$ $0\}$. Note that $w_{i}=C^{*(g-i)} /(g-i)$ !, where by abusing the notations, $C$ denotes 
also its image in $J(C)$, thus $\left(w_{i}\right)_{(s)}=0$ for any $s<0$. This gives the following equations:

(4)

$$
\left\{\begin{array}{c}
\left(b_{1}\right)_{(j)}\left(w_{p}\right)_{(0)}-\left(b_{2}\right)_{(j)}\left(w_{p-1}\right)_{(0)}+\cdots+(-1)^{k}\left(b_{k}\right)_{(j)}\left(w_{p+1-k}\right)_{(0)}=0 \\
\vdots \\
\left(b_{1}\right)_{(j)}\left(w_{g-1}\right)_{(0)}-\left(b_{2}\right)_{(j)}\left(w_{g-2}\right)_{(0)}+\cdots+(-1)^{k}\left(b_{k}\right)_{(j)}\left(w_{g-k}\right)_{(0)}=0
\end{array}\right.
$$

By Poincaré's formula, $C_{(0)}$ has the same cohomological class as $\frac{\Theta^{g-1}}{(g-1) !}$ and the later lies also in $C H_{(0)}^{g-1}(J(C))$. By [B2] (p. 650), the map $C H_{(0)}^{g-1} \rightarrow H^{2 g-2}$ is injective, thus we obtain $C_{(0)}=\frac{\Theta^{g-1}}{(g-1) !}$. By Cor. 2 [B1] (p. 249), we have $(g-r) ! C_{(0)}^{* r}=r ! \Theta^{g-r}$, which gives that $\left(w_{g-r}\right)_{(0)}=\frac{\Theta^{g-r}}{(g-r) !}$ for all $1 \leq r \leq g$. Using these formulae, and noting that $p=g-k-1$, equations (4) give the following

$$
\left\{\begin{array}{c}
\left(b_{1}\right)_{(j)} \Theta^{g-k-1} /(g-k-1) !-\cdots+(-1)^{k-1}\left(b_{k}\right)_{(j)} \Theta^{g-2 k} /(g-2 k) !=0 \\
\vdots \\
\left(b_{1}\right)_{(j)} \Theta^{g-2} /(g-2) !-\cdots+(-1)^{k-1}\left(b_{k}\right)_{(j)} \Theta^{g-k-1} /(g-k-1) !=0
\end{array}\right.
$$

Multiplying the $\mathrm{j}$-th equation in (5) by $\Theta^{k-j}$, we obtain a linear system of equations of $\left(b_{1}\right)_{(j)} \Theta^{g-2}, \cdots,\left(b_{k}\right)_{(j)} \Theta^{g-k-1}$, whose coefficient matrix has nonzero determinant, thus $\left(b_{1}\right)_{(j)} \Theta^{g-2}=0$ in $C H(J(C))$, which implies that $\left(b_{1}\right)_{(j)}=0$ in $C H^{1}(J(C))$. We can now use the first $(k-1)$ equations and a similar argument to deduce that $\left(b_{2}\right)_{(j)} \cdot \Theta^{g-4}=0$, which by our hypothesis implies that $\left(b_{2}\right)_{(j)}=0$. We can continue to obtain that $\left(b_{i}\right)_{(j)}=0$ for all $i$, a contradiction to the definition of $j$.

Remark 4. (i) In general, one expects that if Conjecture 1 holds for a smooth projective variety $X$, then it holds for projective bundles on $X$. Unfortunately, we can only prove this for a few cases (see Proposition 5 and the previous Lemma).

(ii) With some efforts, one can prove in a similar way that the Lemma holds without the restriction on $p$.

Theorem 1. Let $J(C)$ be the Jacobian of a smooth projective curve $C$ and $\Theta$ a symmetric theta divisor. The following conjectures are equivalent:

(i) Beauville's conjecture for $J(C)$;

(ii) Conjecture 1 for $J(C)$ for any ample divisor $D$; 
(iii) Conjecture 1 for $(J(C), \Theta)$;

(iv) Conjecture 1 for all $\left(C^{(k)}, z_{k}\right), k \geq 1$;

(v) Conjecture 1 for $\left(C^{(2 g-1)}, z\right)$.

Proof. The equivalence between (i) and (v) is given by Prop. 7, whose proof also gives the implication $(\mathrm{v}) \Rightarrow$ (iv).

We now prove the implication (i) $\Rightarrow$ (ii). Let $A$ be an abelian variety of dimension $g$ and $D$ an ample divisor. We denote by $\sigma: A \rightarrow A$ the multiplication by -1 . Then $D=D_{0}+D_{1}$ with $D_{0}=\left(D+\sigma^{*} D\right) / 2 \in$ $C H_{(0)}^{1}(A)$ and $D_{1}=\left(D-\sigma^{*} D\right) / 2 \in C H_{(1)}^{1}(A)$. One notices that $D_{0}$ is an ample symmetric divisor on $A$. By the motivic Hard Lefschetz theorem of Künnemann [K], the following map

$$
\cdot\left(D_{0}\right)^{g+s-2 p}: C H_{(s)}^{p}(A) \rightarrow C H_{(s)}^{g+s-p}(A), 0 \leq 2 p-s \leq g
$$

is an isomorphism. Assume $D^{g-2 p} \alpha=0$ for some $\alpha \in C H^{p}(A)$. By (i), we can write $\alpha=\sum_{s=0}^{p} \alpha_{(s)}$ with $\alpha_{(s)} \in C H_{(s)}^{p}(A)$. If $\alpha \neq 0$, let $j=\min \left\{s \mid \alpha_{(s)} \neq 0\right\}$, then the equation $\left(D_{0}+D_{1}\right)^{g-2 p} \alpha=0$ implies that $\left(D_{0}\right)^{g-2 p} \alpha_{(j)}=0$. As $j \geq 0$, we get $\left(D_{0}\right)^{g-2 p+j} \alpha_{(j)}=0$, which implies $\alpha_{(j)}=0$, contradicting to the definition of $j$. This proves (ii).

We now prove (iii) $\Rightarrow$ (i). If $2 p+1 \geq g$, then by Lemma 4 and Proposition 7. $C H_{(s)}^{p}(J(C))=0$ for $s<0$. If $2 p+1<g$, then $2(g-p-1)+1>g$. By Lemma 4, the strong stability holds for $C$ and $g-p-1$. By Lemma 3 , Beauville's conjecture holds for $C H^{p}(J(C))$.

As Beauville's conjecture holds for abelian varieties of dimension $\leq 4$ by [B2], we obtain the following

Corollary 5. Let $C$ be a smooth projective curve of genus $\leq 4$ and $k a$ natural integer. Then Conjecture 1 holds for $\left(C^{(k)}, z_{k}\right)$.

Remark 5. (i). After the first draft of this paper, A. Beauville found another proof of the implication (iii) $\Rightarrow$ (i) in [B3].

(ii). In [S], Soulé proved the map in Conjecture 1 is in fact an isomorphism for abelian varieties defined over a finite field. In this case, Beauville's conjecture can also be proved using Frobenius maps (see remark 3 in [B2]). 


\section{Murre's conjectures}

In [M], Murre proposed the following conjectures on the structure of Chow groups of a smooth projective variety $X$ of dimension $n$ :

(A) there exists a Chow-Künneth decomposition $\Delta_{X}=\sum_{i=0}^{2 n} \pi_{i}$.

(B) $\pi_{0}, \cdots, \pi_{j-1}$ and $\pi_{2 j+1}, \cdots, \pi_{2 n}$ acts as zero on $C H^{j}(X)$.

(C) the filtration defined by $F^{v} C H^{j}(X)=\operatorname{Ker}\left(\pi_{2 j}\right) \cap \operatorname{Ker}\left(\pi_{2 j-1}\right) \cdots \cap$ $\operatorname{Ker}\left(\pi_{2 j-v+1}\right)$ is independent of the choice of $\pi_{i}{ }^{\prime}$ 's.

(D) $F^{1} C H^{j}(X)=C H^{j}(X)_{h o m}$.

It has been proved in [J] that Murre's conjectures (for all $X$ ) are equivalent to the Bloch-Beilinson conjecture. Unfortunately these conjectures are very hard to prove, and only a very few cases are known.

Let $A$ be a $g$-dimensional complex abelian variety and $c_{0}: C H_{(0)}^{p}(A) \rightarrow$ $H^{2 p}(A)$ the cycle map. It is known that Murre's conjecture (A) holds for abelian varieties (see [M] and the references therein). In [B2], it was conjectured that $c_{0}$ is always injective. The following result is well-known and is easily deduced from [M].

Lemma 5. Let $A$ be an abelian variety. Then Murre's conjectures (B) and (D) hold for $A$ if and only if Beauville's conjecture holds for $A$ and the cycle map $c_{0}: C H_{(0)}^{p}(A) \rightarrow H^{2 p}(A)$ is injective for all $p$.

Theorem 2. For abelian varieties, Murre's conjectures (B) and (D) are equivalent to Conjecture 2 .

Proof. Assume Conjecture 2 for abelian varieties, then Conjecture 1 also holds, which implies Beauville's conjecture by Theorem 1. We proceed by induction on $p$ to prove the injectivity of $c_{0}$. The case $p=0$ is trivial. Assume we have proved it for $p-1$ with $p \leq g / 2$. Let $D$ be an ample symmetric divisor on $A$. By [K], the map $\cdot D^{g-2 p+2}: C H_{(0)}^{p-1}(A) \rightarrow C H_{(0)}^{g-p+1}(A)$ is an isomorphism. Together with the Hard Lefschetz theorem on cohomology, this implies the injectivity of the map $C H_{(0)}^{g-p+1}(A) \rightarrow H^{2 g-2 p+2}(A)$. Now for any element $\alpha \in C H_{(0)}^{p}(A)_{h o m}$, the cohomology class of $\alpha D^{g-2 p+1}$ vanishes, which implies that $\alpha D^{g-2 p+1}=0$ in $C H_{(0)}^{g-p+1}(A)$. By Conjecture 2, this gives $\alpha=0$. In other words, the map $C H_{(0)}^{p}(A) \rightarrow H^{2 p}(A)$ is injective.

Assume Murre's conjecture. Then the injectivity of $c_{0}$ and Beauville's conjecture imply $C H^{p}(A)_{h o m}=\oplus_{s \geq 1} C H_{(s)}^{p}(A)$. Now a similar argument as done in the proof of Theorem 1 implies the injectivity of $\cdot D^{g-2 p+1}$ : 
$\oplus_{s \geq 1} C H_{(s)}^{p}(A) \rightarrow \oplus_{s \geq 1} C H_{(s)}^{g-p+1}(A)$ for any ample divisor $D$ on $A$, concluding the proof.

In a similar way as the proof of Lemma 4, one can show that if Murre's conjectures (B) and (D) hold for a Jacobian $J(C)$, then Conjecture 2 holds for $\left(C^{(k)}, z_{k}\right)$. This give the following

Corollary 6. Conjecture Q holds for $\left(C^{(k)}, z_{k}\right)$ for all curves $C$ of genus $\leq 3$.

\section{References}

[B1] Beauville, A., Quelques remarques sur la transformation de Fourier dans l'anneau de Chow d'une variété abélienne, Algebraic geometry (Tokyo/Kyoto, 1982), 238-260, Lecture Notes in Math., 1016, Springer, Berlin, 1983.

[B2] Beauville, A., Sur l'anneau de Chow d'une variété abélienne, Math. Ann. 273 (1986), no. 4, 647-651

[B3] Beauville, A., The action of SL(2) on abelian varieties, arXiv:0805.1541

[B-S] Bloch, S.; Srinivas, V. Remarks on correspondences and algebraic cycles. Amer. J. Math. 105 (1983), no. 5, 1235-1253

[C] Collino, A., The rational equivalence ring of symmetric products of curves, Illinois J. Math. 19 (1975), no. 4, 567-583

[G] Grothendieck, A., Standard conjectures on algebraic cycles, in Algebraic Geometry (Internat. Colloq., Tata Inst. Fund. Res., Bombay, 1968) pp. 193-199 Oxford Univ. Press, London

[F] Fukuma, Y., On polarized surfaces $(X, L)$ with $h^{0}(L)>0, \kappa(X)=2$, and $g(L)=q(X)$, Trans. Amer. Math. Soc. 348 (1996), 4185-4197.

[H] Hartshorne, R., Equivalence relations on algebraic cycles and subvarieties of small codimension, in Algebraic geometry (Proc. Sympos. Pure Math., Vol. 29, Humboldt State Univ., Arcata, Calif., 1974), 129-164. Amer. Math. Soc., Providence, R.I., 1973 
[J] Jannsen, U., Motivic sheaves and filtrations on Chow groups, in Motives (Seattle, WA, 1991), 245-302, Proc. Sympos. Pure Math., 55, Part 1, Amer. Math. Soc., Providence, RI, 1994

[K] Künnemann, K., A Lefschetz decomposition for Chow motives of abelian schemes, Invent. Math. 113 (1993), no. 1, 85-102

[K-V] Kimura, S.-i.; Vistoli, A., Chow rings of infinite symmetric products, Duke Math. J. 85 (1996), no. 2, 411-430

[Ma] Mattuck, A., Symmetric products and Jacobians, Amer. J. Math. 83 1961 189-206

[M] Murre, J., On a conjectural filtration on the Chow groups of an algebraic variety, I. The general conjectures and some examples, Indag. Mathem., N.S. 4(2) (1993), 177-188

[P] Paranjape, K. H., Cohomological and cycle-theoretic connectivity, Ann. of Math. (2) 139 (1994), no. 3, 641-660

[S] Soulé, C., Groupes de Chow et $K$-théorie de variétés sur un corps fini, Math. Ann. 268 (1984), no. 3, 317-345

[V1] Voisin, C., Variations de structure de Hodge et zéro-cycles sur les surfaces générales, Math. Ann. 299 (1994), no. 1, 77-103

[V2] Voisin, C., Remarks on filtrations on Chow groups and the Bloch conjecture, Ann. Mat. Pura Appl. (4) 183 (2004), no. 3, 421-438 